\title{
Pulmonary Arteriovenous Malformations of Late Revelation
}

\author{
Adil ZEGMOUT*, Abdelhalim BOUCAID, Youness AMCHICH, Yassir BENBABA, Hicham \\ SOUHI, Hanane EL OUAZZANI, Ismail Abderrahmane RHORFI and Ahmed ABID
}

Service de Pneumologie, Hôspital Militaire D'Instruction Mohamed V, Rabat, Morocco

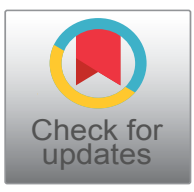

*Corresponding author: Adil ZEGMOUT, Service de Pneumologie, Hôspital Militaire D'instruction Mohamed V, Rabat, Morocco, Tel: (+212)-666744694

\begin{abstract}
Pulmonary arteriovenous malformations are scarce and hard to diagnose. We report a case of a very late discovery at an advanced age. Recent vascular imaging techniques (Three-dimensional Computed Tomographic Angiography) have largely contributed to their diagnosis. The patient is treated with arterial embolization which is effective and carries a low complication rate in skilled hands. This case illustrates the clinical circumstances of diagnosis, the diagnostic algorithm, and the differential diagnosis to be discussed in this radio-clinical presentation, as well as the peculiarity of thinking about this malformation despite the advanced age.
\end{abstract}

\section{Keywords}

Pulmonary arteriovenous malformation, Three-dimensional computed tomographic angiography, Embolization

\section{Abbreviations \\ PAVM: Pulmonary Arteriovenous Malformations}

\section{Introduction}

Pulmonary arteriovenous malformations (PAVM) are rare vascular abnormalities that are difficult to diagnose. Congenital PAVM are exceptionally discovered in adulthood [1]. This observation is a reminder of the circumstances of discovery of this very late-onset vascular abnormality, and the elements that led to its diagnosis.

\section{Observation}

Aged of 56 years, Chronic smoker with no known medical history and no significant family history. The patient reports chronic dyspnea associated with low hemoptysis. Clinical examination did not find any abnormality. The Chest X-ray shows a well limited left parac- ardiac rounded opacity.

The etiologies evoked by this radio-clinical presentation are primarily infectious (pulmonary tuberculosis, pneumonia), pulmonary embolism and pulmonary neoplasia, especially in light of age and a history of chronic smoking, these causes have been eliminated by the absence of biological inflammatory syndrome, negative D-Dimeres, a negative quantiferon and the searches for mycobacteria in the sputum and bronchial aspiration liquid which were negative with sterile cultures, a genexpert did not detect Mycobacterium tuberculosis.

The chest CT scan did not reveal any lesional process, but did show a varicose appearance involving the antero-basal segment of the left lower lobe of vascular type, causing the pulmonary vein to communicate distally with the pulmonary artery (Figure 1). A thoracic angioscan with arterial and venous time, and a three-dimensional reconstruction was performed, confirmed the diagnosis of MAVP, showing an abnormally dilated aspect of the left lower lobe pulmonary vein, measured at 5 $\mathrm{mm}$, who's followed to its distality leads to a sacciform formation, extended over $17 \mathrm{~mm}$, with venous return, measured at $2.5 \mathrm{~mm}$ and communicating with the lower lobar of the left pulmonary artery (Figure 2).

Ambient air arterial gasometry shows hypoxemia with $\mathrm{PaO}_{2}$ at $68 \mathrm{mmHg}, \mathrm{PCO}_{2}$ at $43 \mathrm{mmHg}, \mathrm{pH}$ at 7.38 . Echocardiography and plethysmography are normal. Because of the uniqueness of this AVMP and the negativity of the etiological analyses, the diagnosis of congenital AVMP was retained.

After multidisciplinary discussion, the indication for 


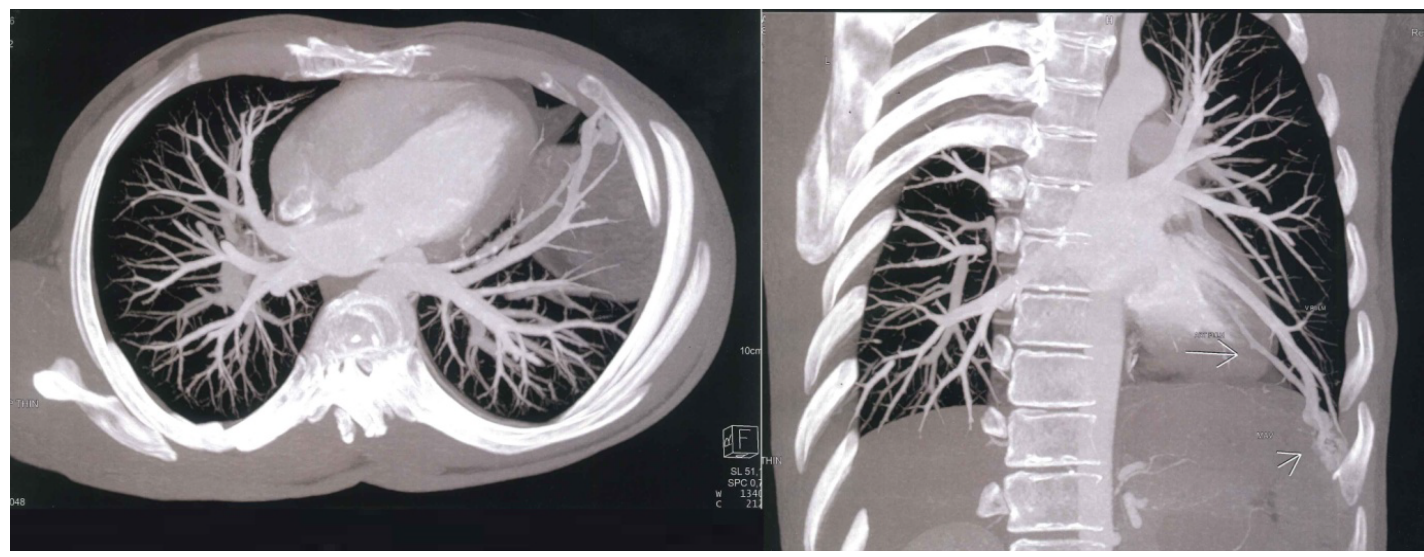

Figure 1: CT scan showing the unusual arteriovenous communication in the left lower lobe (arrow) between the antero-basal pulmonary artery trunk and the lower pulmonary vein.
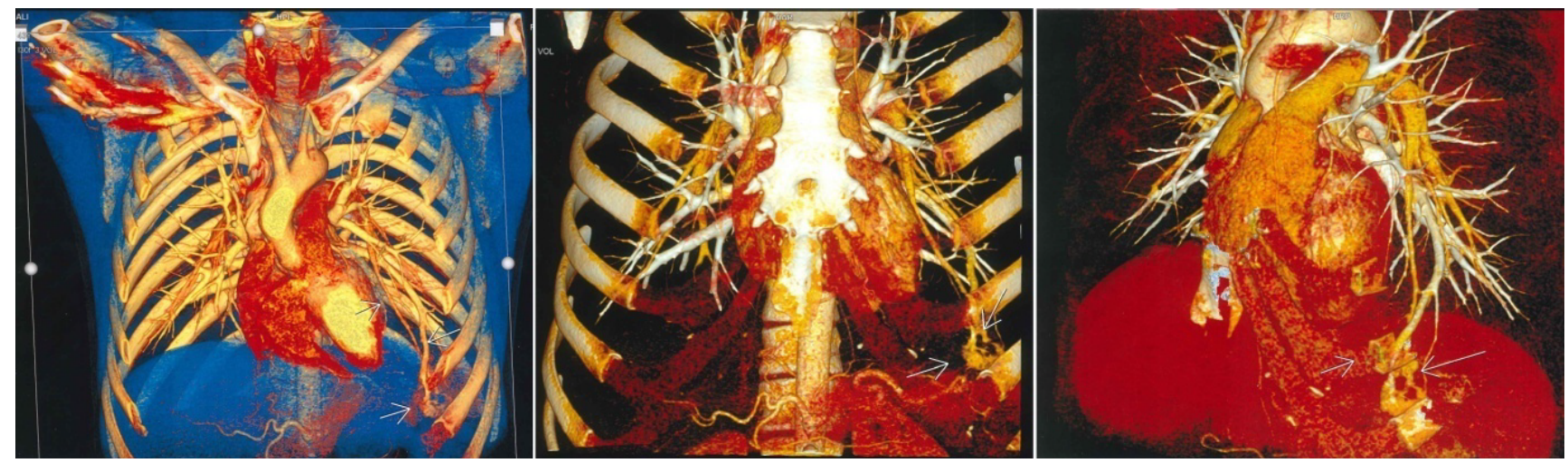

Figure 2: Three-dimensional reconstruction of the frontal angioscanner of the arteriovenous malformation of the antero-basal seat of the left lower lobe (arrows).

arterial embolisation was retained despite the absence of functional signs. The arguments for this action were the potential long-term complications of chronic hypoxemia, the risk of infection and the risk of hemorrhage. Since then, the patient has been asymptomatic without recurrence of the symptomatology.

The patient was placed on extencillin-based antibiotic prophylaxis, follow-up was carried out by clinical examination and CT scan, and no complications or recurrences were detected.

\section{Discussion}

Pulmonary Arteriovenous Malformations are rare entities with an estimated rate of 3-5 cases per 100,000 inhabitants [2]. They are defined by direct communication between the pulmonary arterial and venous systems, creating a right-left shunt [3]. They can be congenital (Rendu-Osler disease) or acquired. The age of discovery is highly variable, from the neonatal period to adulthood (more frequent between 30 and 40 years of age) [1]. Our observation is distinguished by a very late age of revelation of the malformative pathology, which was very well supported clinically.

The clinical manifestations are dominated by exertional dyspnea, which is not always correlated with the size of the shunt [4]. This symptom, although frequent, is non-specific and common to many pulmonary pathologies. Our patient has linked chronic dyspnea to the complications of smoking and sedentary lifestyle. Hemoptysis is quite rare but sometimes massive.

However, in our context, tuberculosis is the first etiology to evoke in front of a haemoptysis with pulmonary opacity, in order to eliminate this diagnosis it is necessary to make repeated bacteriological investigations on sputum as well as on bronchial aspiration, this procedure will delay the exact diagnosis.

The complications can be severe and sometimes fatal. Unfortunately, they are often the mode of revelation of the PAVM. The most common complications affect the central nervous system and occur in approximately $30 \%$ of the patients ( $70 \%$ of patients with diffuse forms of PAVM) [5], these are stroke, transitory ischemic stroke mechanisms, brain abscesses [6-8]. Also the authors note that the PAVM are also associated with migraine headaches in particular with aura.

Other complications are rarer but potentially lethal; they are dominated by the hemothoraxes, which are secondary to MAVP rupture [9].

The chest X-rays are insufficient to detect these ab- 
normalities, if visible they take the form of a round or oval image of uniform density with well defined edges. The helical multislice chest CT is the gold standard and allows visualization of the anevrysmal sac and associated efferent vessels [2]. The sensitivity of the scanner is higher than the conventional pulmonary angiography (98\% vs. $60 \%$ ) [10]. In our observation, the 3-D helical scanner was able to clarify the angio-architecture of the Arteriovenous Malformations.

Embolization by percutaneous closure of the associated arteries has replaced surgical removal, limiting the surgical interventions to very large volume and perihilar malformations or in case of embolization failure [6].

Prevention of complications related to PAVM includes antibiotic prophylaxis in the event of actions that can lead to bacteremia (dental care, endoscopies, surgical procedures, embolization of PAVM...), despite the absence of a randomized study. The protocol of antibiotic prophylaxis is modelled on that recommended for prevention of bacterial endocarditis in patient's carriers of cardiac valvulopathy [11].

Also the continuation of antibiotic prophylaxis is imperative after embolisation because of the possibility of persistent or recurring microfistulas of MAVP $[6,12]$.

\section{Conclusion}

Although Pulmonary Arteriovenous Malformations have been described for a long time, our observation seemed original to us by its very late revelation. The authors also recall the non-specific clinical presentation, which was much more suggestive of tuberculosis in our context or lung cancer explaining the diagnostic difficulties.

\section{Conflicts of Interest}

None.

\section{Statement of Equal Author's Contribution}

I declare that the authors have attributed to the manuscript.

\section{References}

1. Delacourt C, Remy-Jardin M, Regnard JF, Piégay F (2011) Lung malformations discovered in adulthood. Revue des Maladies Respiratoires Actualités 3: 162-166.

2. Blivet S, Lacombe P, Chinet T (2011) Malformations artério-veineuses pulmonaires. Revue des maladies respiratoires Actualités 3: 61-66.

3. Gossage JR, Kanj G (1998) Pulmonary arteriovenous malformations. A State of the art review. Am J Respir Crit Care Med 158: 643-661.

4. Swanson KL, Prakash UB, Stanson AW (1999) Pulmonary arteriovenous fistulas: Mayo clinic experience, 1982-1997. Mayo Clini Proc 74: 671-680.

5. Faughnan ME, Lui YW, Wirth JA, Pugash RA, Redelmeier DA, et al. (2000) Diffuse pulmonary arteriovenous malformations: Characteristics and prognosis. Chest 117: 31-38.

6. Trerotola SO, Pyeritz RE (2010) PAVM embolisation: An update. AJR Am J Roentgenol 195: 837-845.

7. Moussoutas M, Fayad P, Rosenblatt M, Hashimoto M, Pollak J, et al. (2000) Pulmonary arteriovenous malformations: Cerebral ischemia and neurologic manifestations. Neurology 55: 959-964.

8. Maher CO, Piepgras DG, Brown RD, Friedman JA, Pollock BE (2001) Cerebrovascular manifestations in 321 cases of hereditary hemorrhagic telangiectasia. Stroke 32: 877-882.

9. Faughnan ME, Granton JT, Young LH (2009) The pulmonary vascular complications of hereditary haemorrhagic telangiectasia. Eur Respir J 33: 1186-1194.

10. Cottin V, Blanchet AS, Cordier JF (2006) Manifestations pulmonaires de la maladie de rendu-osler: Dépistage, diagnostic et traitement. Rev Mal Respir 23: 4853-4866.

11. Faughnan ME, Palda VA, Garcia-Tsao G, Geisthoff UW, McDonald J, et al. (2011) International guidelines for the diagnosis and management of hereditary hemorragic telangiectasia. J Med Genet 48: 73-87.

12. Lee WL, Graham AF, Pugash RA, Hutchison SJ, Grande P, et al. (2003) Contrast echocardiography remains positive after treatment of pulmonary arteriovenous malformations. Chest 123: 351-358. 